\title{
REPRESENTAÇÕES SOCIAIS DA EQUIPE DE ENFERMAGEM SOBRE OS CUIDADOS DURANTE A HIGIENIZAÇÃO NO PACIENTE IDOSO
}

NURSING TEAM'S SOCIAL REPRESENTATIONS ABOUT CARE WHEN PROVIDING ELDERLY PATIENTS WITH HYGIENE

REPRESENTACIONES SOCIALES DEL EQUIPO DE ENFERMERÍA ACERCA DEL CUIDADO DURANTE LA HIGIENIZACIÓN DEL PACIENTE

ANCIANO

Thaynara Ferreira Lopes 1

Bruna Karen Cavalcante Fernandes 2

Jéssica de Menezes Nogueira ${ }^{3}$

Maria Célia de Freitas 4

Palavras-chave:

Enfermagem; Idoso; Cuidados de Enfermagem; Pele.

Keywords:

Nursing; The Elderly; Nursing Care; Skin.

Palabras clave: Enfermería; Anciano; Atención de Enfermería; Piel.

Submetido: 09/12/2019

Aprovado: 06/05/2020

Autor(a) para Correspondência: Thaynara Ferreira Lopes Av. Dr. Silas Munguba, 1700 Itaperi, Fortaleza - CE CEP: 62042-030

E-mail: thaynaralopes.13@ hotmail.com

\section{RESUMO}

Este estudo teve por objetivo apreender as representações sociais da equipe de enfermagem sobre os cuidados dedicados ao idoso durante a higiene corpórea. Trata-se de estudo descritivo com abordagem qualitativa. Os dados foram coletados por entrevistas semiestruturadas com participação de 6 enfermeiros e 16 técnicos de enfermagem da equipe de um hospital de atendimento terciário localizado em Fortaleza-CE. Para o processamento dos resultados, recorreu-se ao software ALCESTE (Analyse Lexicale par Contexte d'un Ensemble de Segments de Texte), versão 2012. Adotou-se como método a análise do conteúdo, desenvolvida com base na Teoria das Representações Sociais (TRS) de Serge Moscovici. Partiu-se da análise da classe 3, que ressalta a prevenção de complicações, e da classe 4, que reforça a importância dos cuidados com a pele do idoso, bem como quais tecnologias são necessárias para isso. Foi possivel identificar os temas mais citados pelos profissionais em relação à higiene do idoso, aos principais cuidados prestados e à importância dos cuidados com a pele do idoso.

1. Enfermeira graduada pela Universidade Estadual do Ceará (UECE). E-mail: thaynaralopes.13@hotmail.com ORCID: https://orcid.org/0000-0003-4974-7693

2. Enfermeira. Doutoranda em Cuidados Clínicos em Enfermagem e Saúde na UECE. E-mail: brunnakaren@hotmail. com ORCID: https://orcid.org/0000-0003-2808-7526

3. Enfermeira. Doutora em Cuidados Clínicos em Enfermagem e Saúde pela UECE. E-mail: jessicademenezesn@ hotmail.com ORCID: https://orcid.org/0000-0002-8201-7169

4. Enfermeira. Professora no Curso de Enfermagem da UECE. E-mail: celfrei@hotmail.com ORCID: https://orcid. org/0000-0003-4487-1193 


\section{ABSTRACT}

This study aimed to grasp nursing team's social representations about the care provided to the elderly during body hygiene. This is a descriptive study with a qualitative approach. Data was collected through semi-structured interviews conducted with 6 nurses and 16 nursing technicians from the staff of a tertiary care hospital located in Fortaleza, Ceará, Brazil. For the processing of results, the software ALCESTE (Analyse Lexicale par Contexte d'un Ensemble de Segments de Texte), version 2012, was used. Content analysis was adopted as a method, having Serge Moscovici's Theory of Social Representations (TSR) as a basis. The study started from the analysis of class 3, which emphasizes the prevention of complications, and class 4, which reinforces the importance of skin care for the elderly, as well as which technologies are needed to do this. The approach managed to identify the themes most frequently cited by practitioners in relation to hygiene for the elderly, the main patient care actions taken, and the importance of skin care for the elderly.

\section{RESUMEN}

Este estudio tuvo como objetivo aprehender las representaciones sociales del equipo de enfermería acerca de la atención dedicada al anciano durante la higiene corporal. Este es un estudio descriptivo con un enfoque cualitativo. Los datos fueron recolectados a través de entrevistas semi-estructuradas con participación de 6 enfermeros y 16 técnicos de enfermería del equipo de un hospital de atención terciaria ubicado en Fortaleza, Ceará, Brasil. Para el procesamiento de los resultados, se utilizó el software ALCESTE (Analyse Lexicale par Contexte d'un Ensemble de Segments de Texte), versión 2012. El análisis de contenido se adoptó como método, desarrollado en base a la Teoría de las Representaciones Sociales (TRS) de Serge Moscovici. Se inició con el análisis de la clase 3, que destaca la prevención de complicaciones, y de la clase 4, que refuerza la importancia del cuidado de la piel del anciano, así como qué tecnologías se necesitan para esto. Fue posible identificar los temas más citados por los profesionales con relación a la higiene del anciano, a los principales cuidados brindados y a la importancia del cuidado de la piel para el anciano.

\section{INTRODUÇÃ $O$}

0 envelhecimento populacional no Brasil cresce de forma acelerada devido aos melhores cuidados de saúde à população idosa, valorizando-a e oferecendothe melhor cuidado, com o consequente aumento da expectativa de vida. Segundo o Ministério da Saúde (MS), as estatísticas da Organização Mundial da Saúde (OMS) têm como estimativa para o período 1950-2025 que o número de idosos no país aumente 15 vezes, e a população geral, 5 vezes. Em 2025, - Brasil poderá registrar cerca de 32 milhões de pessoas com 60 anos ou mais ${ }^{1}$.

Em nível mundial, o número global de idosos está projetado para aumentar de 962 milhões, em 2017, para 1,4 bilhão, em 2030, e 2,1 bilhões em 2050, quando todas as regiões do mundo, exceto a África, terão $1 / 4$ ou mais de sua população com 60 anos de idade ou mais ${ }^{2}$.

A avaliação clínica do idoso deve ser criteriosa, para que se possa traçar um plano de cuidados, de prevenção de danos e/ou para impedir a piora de algum problema identificado. Assim, o enfermeiro assume o protagonismo nesse âmbito, dada a sua importância na promoção e na prevenção de agravos à saúde da população idosa, valendo-se do raciocínio clínico voltado à assistência ao idoso.

Um dos aspectos do olhar clínico do enfermeiro ao idoso está relacionado à condição geral da pele, órgão por meio do qual os microrganismos podem penetrar no organismo, causando doenças e infecções. A pele também é responsável por vários fatores de equilíbrio e proteção à saúde, razão pela qual se mostra necessária uma abordagem geral sobre a fisiologia da pele e sobre as formas de higiene corpórea, com foco na pele do idoso e em suas alterações patológicas, o que fundamenta uma proposta de pesquisa em enfermagem. Para tanto, compreender como a equipe de enfermagem põe em prática o processo de higiene corpórea se mostra crucial para aprofundar o entendimento de como a equipe de enfermagem lida com o cuidar do corpo e da pele dos pacientes ${ }^{3}$.

Diante de tal quadro, utiliza-se o arcabouço teórico-metodológico da Teoria das Representações Sociais (TRS) de Serge Moscovici, a qual se apresenta 
como uma das alternativas dessa nova perspectiva de compreensão do processo saúde-doença, uma vez que ela tenta compreender, a mesmo tempo, a dimensão cognitiva e social desse fenômeno ${ }^{4}$. Nesse contexto, a equipe de enfermagem é importante para fazer a higiene corpórea e as intervenções preventivas de agravos à saúde do paciente, além de manter a integridade da pele do idoso.

Ademais, pode-se estabelecer um modelo de cuidado que permeia as mudanças próprias do envelhecimento, associadas à sua experiência de vida e, com isso, propor um plano de cuidados que considere seu contexto de saúde e doença, proporcionado de forma humana com base em uma abordagem integral ${ }^{5}$. Também é importante valorizar a individualidade do paciente, vislumbrando uma assistência de qualidade, pautada em uma relação empática e holística.

Nesse contexto, compete à equipe de saúde, incluindo o enfermeiro, dar apoio à saúde do idoso, ofertando-the cuidados que possibilitem promover sua saúde, prevenir doenças e agravos à saúde, bem como alívio dos sintomas da doença e manutenção da independência e da autonomia para realizar suas atividades diárias. As ações de enfermagem incluem atenção integral ao paciente e à família, o que favorece que esta acompanhe e esteja ao lado dele sempre que necessário ${ }^{6}$.

Assim, este estudo teve por objetivo apreender as representações sociais da equipe de enfermagem sobre os cuidados dedicados ao idoso durante a higiene corpórea.

\section{METODOLOGIA}

Trata-se de estudo descritivo e analítico, com abordagem qualitativa, que faz emergir aspectos novos e com capacidade de investir profundamente na análise dos significados e, além de estar sob a perspectiva do sujeito, evidencia o universo de significados, crenças, hábitos, costumes e relacionamentos do paciente. Enfim, enfoca um nível de realidade em que a abordagem qualitativa é o modelo de escolha, apoiando-se na TRS ${ }^{4}$.

0 estudo da TRS deve responder 3 questões básicas: 1) Quem sabe e de onde sabe? Neste estudo, os participantes são os profissionais da equipe de enfermagem que trabalham em um hospital de nível terciário em Fortaleza-CE, cujo público é majoritariamente composto pela população idosa; 2) 0 quê e como se sabe? Essa questão visa a tratar

\section{...compete à \\ equipe de saúde, incluindo o enfermeiro, dar apoio à saúde do idoso...}

dos processos e estados das representações. Logo, o conteúdo, as informações, as fontes e os processos de construção da representação da higiene corporal são focos para respondê-la; e 3) Sobre o que se sabe e com qual efeito? Essa questão se relaciona, em particular, ao estatuto epistemológico das representações sociais, relações que estas mantêm com o conhecimento científico e com a própria realidade que as origina, fazendo com que sejam compreensíveis as ações dos sujeitos diante do objeto, que, no caso, é a higiene corporal do idoso?

0 estudo foi conduzido com 6 enfermeiros e 16 técnicos de enfermagem de um hospital no Município de Fortaleza, escolhidos aleatoriamente. A coleta foi feita entre maio e julho de 2016, segundo estes critérios de elegibilidade: enfermeiros e técnicos de enfermagem que trabalham na unidade que atende à população idosa.

0s participantes responderam uma entrevista semiestruturada, com perguntas sobre: a) os cuidados prestados durante o banho no leito; b) os materiais empregados nesse procedimento; c) quais ações de enfermagem eram realizadas antes de iniciar o banho no leito; d) quais cuidados eram dedicados aos pacientes idosos; e e) como os cuidados influenciam a manutenção da integridade da pele, além de quais sentimentos são mobilizados nesse processo. Todas as respostas foram gravadas, com autorização do participante, ou foram anotadas pelo pesquisador e posteriormente transcritas na íntegra.

Depois da transcrição das falas e da imersão, o material discursivo dos sujeitos foi submetido a um tipo particular de análise do conteúdo, a análise lexical, com auxílio do software ALCESTE (Analyse Lexicale par Contexte d'un Ensemble de Segments de Texte), versão 2012, que viabiliza a análise de dados textuais ou de dados estatísticos, verificando a principal informação disponível no texto ${ }^{8}$. Esse tipo de processamento possibilita uma análise processual do fenômeno mediante a aglutinação de contextos que favorecem a determinação de um campo comum 
de representações compartilhadas entre os participantes, organizando e formatando o pensamento social e o pensamento coletivo. 0 programa analisa o corpus e agrega as unidades formadoras de sentidos relacionadas ao mesmo contexto. Na análise deste estudo se destacaram as classes 3 e 4, pois foram as que evidenciaram os cuidados com a pele e as formas de prevenir a quebra da barreira cutânea.

Foram seguidos todos os preceitos éticos em pesquisas com seres humanos, conforme a Resolução $n$. 466/2012, do Conselho Nacional de Saúde (CNS), do MS9 0 estudo foi aprovado pelo Comitê de Ética em Pesquisa da Universidade Estadual do Ceará (CEP-UECE). Para manter o anonimato, os participantes foram identificados pela letra $P$ (participante) seguida pela numeração em ordem crescente (P1, P2, P3...).

\section{RESULTADOS E DISCUSSÃO}

0 software ALCESTE processou o corpus com um aproveitamento de $76 \%$, sendo constituído por 22 Unidade de Contexto Inicial (UCI) referentes à quantidade de sujeitos participantes do estudo. Em seguida, o software dividiu o corpus em 409 Unidade de Contexto Elementar (UCE), obtendo 16.200 formas distintas ou palavras diferentes, com 12.154 ocorrências. Posteriormente, o software reduziu os vocábulos às suas raízes, originando 348 palavras analisáveis e 223 palavras suplementares (artigos e pronomes, dentre outras). Tendo em vista essa análise, o programa subdividiu as UCE em 4 classes.

A classificação hierárquica descendente (CHD) se encerra com a formação de 4 classes estáveis, constituídas por UCE com vocabulários semelhantes. A Figura 1 ilustra essas repartições a partir da CHD. A análise dos dados foi feita a partir do grupo de pertença relevado pela CHD. Para este estudo, optou-se por aprofundar a análise das classes 3 e 4, que ressaltaram a importância dos cuidados durante a higienização corpórea e a prevenção da quebra da barreira cutânea no idoso e quais tecnologias são necessárias para obter esse resultado.

\begin{tabular}{|lc|}
\hline Présence & $\Phi(\mathrm{PnI})$ \\
\hline mudanca & 0,45 \\
decubito & 0,42 \\
filme & 0,38 \\
umidade & 0,34 \\
transparenti & 0,34 \\
pneumatico & 0,32 \\
uso & 0,29 \\
duas & 0,29 \\
evit & 0,28 \\
preven & 0,28 \\
colchoes & 0,26 \\
mercado & 0,24 \\
articulado & 0,24 \\
barreira & 0,23 \\
mant & 0,21 \\
ide & 0,18 \\
redobr & 0,18 \\
integridade & 0,18 \\
usar & 0,18 \\
creme & 0,18 \\
\hline Absence & $\Phi($ PnI $)$ \\
\hline banh & $-0,17$ \\
as & $-0,15$ \\
fic & $-0,12$ \\
agua & $-0,11$ \\
idos & $-0,10$ \\
\hline
\end{tabular}

\begin{tabular}{|c|c|}
\hline Présence & $\Phi(\mathrm{PM})$ \\
\hline regi & 0,43 \\
\hline escara & 0,40 \\
\hline sacra & 0,35 \\
\hline enfermeir & 0,35 \\
\hline colchao & 0,33 \\
\hline curativo & 0,32 \\
\hline nas & 0,32 \\
\hline calcaneos & 0,31 \\
\hline proemin & 0,27 \\
\hline fazendo & 0,25 \\
\hline ferimento & 0,25 \\
\hline tipo & 0,24 \\
\hline press & 0,24 \\
\hline produto & 0,23 \\
\hline coloc & 0,22 \\
\hline ulcer & 0,22 \\
\hline Osseas & 0,22 \\
\hline massagem. & 0,22 \\
\hline 05 & 0,20 \\
\hline edema & 0,17 \\
\hline Absence & $\Phi(\mathrm{PPl})$ \\
\hline cuid & $-0,15$ \\
\hline gente & $-0,12$ \\
\hline paciente & $-0,11$ \\
\hline$d a$ & $-0,09$ \\
\hline ne & $-0,08$ \\
\hline
\end{tabular}

Figura 1 - Classificação das classes 3 e 4 do corpus ALCESTE 2012. Fortaleza, 2018. Fonte: Elaborada pelas autoras. 
A classe 3 se revelou composta pelos julgamentos dos profissionais sobre a melhor forma de prevenir as lesões de pele, julgando a forma como a teoria o preconiza e o que se pratica na assistência prestada pela equipe de enfermagem.

0 s assuntos mais citados na classe 3 se referiram aos cremes de barreira, aos cuidados com os lençóis no leito, à mudança de decúbito, às massagens na pele, ao tipo de colchão usado e aos cuidados durante o banho no leito, como ilustram as falas:

É necessário não deixar nenhuma dobra de lençol, sempre tentar mudar um pouquinho de decúbito e sempre de duas em duas horas fazer a mudança de decúbito, apesar de esses nossos colchões já massagearem a pele. (P5) [...] cobrar mais a questão da hidratação, porque vai melhorando a questão do turgor da pele, a questão da mudança do decúbito em si, porque nós não temos um protocolo de quantas horas para a mudança de decúbito e isso contribui, apesar de usarmos o colchão pneumático e do fato que o colchão não vai evitar que o idoso venha a ter a lesão. (P10)

$\mathrm{Na}$ classe 4 , os sujeitos do estudo relataram como se mantém a integridade da pele do idoso e qual é a função da equipe de enfermagem no processo de prevenção das lesões de pele, mesmo trabalhando com recursos reduzidos, o que causa dualidade de sentimentos entre o que é preconizado na teoria e o que ocorre na prática, como indicam as falas:

Fazemos os curativos, então muitos saram logo, pois têm os produtos adequados para o tipo de escara. Muitas dessas lesões o idoso já chega aqui na unidade com elas, pois a maioria passa muito tempo na emergência utilizando um colchão duro e sem nenhuma proteção. (P3)

Às vezes não tem um hidratante para ajudar e o sabão utilizado resseca a pele deles. Assim, usamos a clorexidina porque não tem outro. A enfermeira faz os curativos e coloca os óleos necessários para melhorar a pele e evitar as lesões. (P4)

Os profissionais enfatizam a importância de assegurar a proteção de determinadas regiões do corpo propensas à formação de lesões. Como, devido ao processo do envelhecimento, a pele do idoso se

\section{Os profissionais \\ enfatizam a importância de assegurar a proteção de determinadas regiões do corpo propensas à formação de lesões.}

mostra fragilizada, com perda do turgor, redução da vascularização e alterações morfológicas, como a desorganização das fibras colágenas e elásticas, aumenta a probabilidade do desenvolvimento de lesões.

Ao considerar uma série de fatores ambientais relativos à idade e inúmeros fatores que a institucionalização do idoso acarreta para uma eficiente barreira de hidratação da pele, tem-se o aumento do risco da quebra da integridade da pele ${ }^{10}$.

No ambiente hospitalar, em geral, o idoso está acamado em decorrência da própria velhice e de sua condição clínica de saúde, fator que potencializa a quebra da integridade da pele. A imobilidade no leito leva ao aumento da pressão em determinadas regiões do corpo do idoso ${ }^{11}$. Diante disso, os profissionais relataram a importância de usar os cremes de barreira, que devem ser aplicados suavemente, com frequente limpeza da pele usando água morna e um produto de limpeza neutro, e não com sabonete, pois este provoca ressecamento da pele. Essas intervenções, como a aplicação de hidratante e o exame da pele durante o banho ou a troca de fralda, por exemplo, indicam a necessidade de estar atento e identificar precocemente os fatores de risco para o desenvolvimento de lesões.

Além disso, os profissionais também afirmaram que:

Não se deve deixar nenhuma dobra de lençol, sempre tentar mudar um pouco de decúbito e sempre de duas em duas horas fazer a mudança de decúbito. (P1)

Assim, é necessário ter cuidado com travesseiros, colchões e outros materiais para que não ocorram pregas, sobretudo naqueles destinados aos pacientes restritos ao leito, pois isso compromete, ainda mais, a condição da pele deles. Já para aqueles cuja integridade da pele corre mais risco de romper-se com facilidade durante o reposicionamento, o cuidado deve ser a prevenção da ocorrência de fricção ${ }^{12}$. Como 
consequência do uso de lençóis com pregas, também pode ocorrer a fricção sobre o tecido cutâneo do idoso, ou seja, o atrito resultante do contato direto de uma superfície, ou um corpo, sobre outra(o). Essa pressão constitui um fator de risco ligado à duração e à intensidade do contato, que alguns autores consideram uma força ou energia física que age perpendicularmente à pele como resultado da gravidade, causando um achatamento tecidual entre dois planos, um pertencente ao paciente e outro externo a ele, sendo, portanto, um fator de risco para o desenvolvimento de uma lesão $0^{13}$.

Outro cuidado importante feito pela equipe de enfermagem, e bastante citado nas entrevistas, foi a mudança periódica de decúbito, pois a pressão prolongada sobre a pele do paciente idoso, principalmente sobre as proeminências ósseas, constitui um grave fator de risco para o desenvolvimento de uma lesão, devido à relação com o cisalhamento constante sobre a pele ${ }^{12}$.

Com isso, uma importante medida de intervenção da enfermagem é reduzir o tempo e a quantidade de pressão a que o paciente está exposto, com mudanças de posição em horários programados. No entanto, sua frequência deve considerar a condição do paciente (tolerância tecidual, nível de atividade e mobilidade) e a superfície de apoio em uso, para que não ocorram lesões.

0 suprimento sanguíneo deficiente é umas das alterações presentes na pele do idoso, pois, com o avanço da idade, ocorrem alterações na vascularização da pele, além de diminuição do leito vascular com fragilidade dos vasos sanguíneos, causando redução no suprimento tecidual, palidez e alterações na termorregulação. Assim, uma importante intervenção de enfermagem é a massagem de conforto, caracterizada pela manipulação manual e sistemática dos tecidos corporais, transmitindo ao paciente uma sensação física agradável. Com sua aplicação, estimula-se a circulação sanguínea, proporcionando melhor nutrição dos tecidos e oxigenação mais aprimorada ${ }^{13-14}$.

A relevância do uso de colchões com alternância de pressão se deve ao auxílio na mudança contínua das pressões exercidas nas proeminências ósseas, que aliviam a irrigação sanguínea, reduzindo a possibilidade de isquemia na área ${ }^{13}$. Portanto, diminuir ou aliviar a pressão é a medida profilática mais importante e pode ser alcançada pelo posicionamento adequado do paciente no leito e pelo uso de dispositivos redutores da pressão.

\section{...diminuir ou \\ aliviar a pressão \\ é a medida \\ profilática mais \\ importante...}

Além disso, umas das tecnologias mais empregadas atualmente com o paciente hospitalizado é o colchão pneumático, cujo objetivo é proporcionar conforto e proteger sua pele contra a formação de lesões. A pele do paciente idoso hospitalizado apresenta alterações em todos os seus componentes, causando diminuição das camadas, da elasticidade e da resistência, alentecendo o processo cicatricial, aumentando a incapacidade de proteção contra fatores mecânicos, provocando maior fragilidade capilar e reduzindo a perfusão e a nutrição dos tecidos ${ }^{15-16}$.

Portanto, deve-se assegurar o uso de colchões com superfície redistribuída de pressão, o que reduz a probabilidade de formação de novas lesões. Além da ação preventiva, esse colchão também garante uma sustentação mais confortável do corpo. Há no mercado modelos que adotam pressão alternante entre o corpo e o colchão, pela insuflação e desinflação de ar comprimido em bolsões pneumáticos ${ }^{16}$.

Os participantes também mencionaram a importância do cuidado durante o banho no leito, quando há oportunidade de: a) verificar a integridade da pele do paciente; b) observar a necessidade de usar algum creme de barreira; c) avaliar a nutrição, o turgor e a elasticidade da pele; e d) massagear a pele, para propiciar maior conforto e melhorar a circulação. Esse é o momento para fortalecer a relação interpessoal com o paciente, ouvindo suas queixas e seus desconfortos, além de tentar diminuir os sentimentos de ansiedade e tristeza ${ }^{17}$.

0 s profissionais relataram que a manutenção da integridade de pele é feita mediante os cuidados de enfermagem: a) mudança de decúbito; b) verificação de pregas nos lençóis no leito; c) uso de colchões que alternam a pressão; d) massagem de conforto para estimular a circulação; e) aplicação de cremes de barreira; e f) banho no leito. Contudo, ficou bastante evidente a dificuldade de implementar a prevenção efetiva devido aos reduzidos recursos da instituição, como falta de cremes, colchões com alternância de pressão e outros materiais necessários.

0 estudo teve como limitação a resistência, por parte de alguns profissionais da equipe de 
enfermagem, em responder os questionamentos sobre o tema da pesquisa. No entanto, os resultados encontrados podem fundamentar a condução de futuros estudos que ampliem a discussão sobre esse tema.

A equipe de enfermagem desempenha um papelchave na promoção e na prevenção de agravos à saúde do paciente idoso institucionalizado, sendo o enfermeiro o profissional de referência para cuidar e prevenir os agravos à pele do idoso. Por isso, é essencial desenvolver competências e ter conhecimento sobre as características do processo de envelhecimento e sobre a prevenção da ocorrência de problemas de saúde entre esses pacientes. Assim, considera-se importante compreender as concepções dos profissionais de enfermagem envolvidos no cuidado, para conhecer as principais ações de enfermagem na higienização corpórea do paciente idoso.

\section{CONCLUSÃO}

Com este estudo, pôde-se identificar que os temas mais citados pelos profissionais de enfermagem, relativos ao cuidado da pele do idoso, foram: a) cremes de barreira; b) lençóis no leito; c) mudança de decúbito; d) massagens na pele; e) tipo de colchão usado; e f) banho no leito.

Portanto, as representações sociais de profissionais de enfermagem podem fornecer subsídios para a elaboração de estratégias de qualificação da equipe. Uma ampla compreensão do idoso torna necessária a implementação de ações educativas que priorizem esse entendimento e o preparo do profissional, para proporcionar um cuidado que estimule a autonomia e previna complicações. Também se constatou a relevância do apoio institucional, mediante atualização de equipamentos, tecnologias e materiais, o que viabiliza a aplicação de recursos à realidade prática.

\section{CONTRIBUIÇÃO DAS AUTORAS}

Thaynara Ferreira Lopes contribuiu com a realização da pesquisa, o delineamento do estudo e a redação do manuscrito. Bruna Karen Cavalcante Fernandes e Jéssica de Menezes Nogueira contribuíram com o delineamento do estudo e a redação do manuscrito. Maria Célia de Freitas contribuiu com o delineamento do estudo e a revisão crítica do manuscrito.

\section{REFERÊNCIAS}

1. Instituto Brasileiro de Geografia e Estatística. Síntese de indicadores sociais. Rio de Janeiro: IBGE; 2014.

2. Organização Mundial da Saúde. Resumo: Relatório Mundial de Envelhecimento e Saúde. Genebra: OMS: 2018.

3. Carvalho PS, Aguiar ESS, Brito KKG, Antas EMV, Andrade SSC, Silva MA, et al. Conhecimento de cuidadores formais de idosos para manter a pele do idoso livre de lesão por pressão. Enfermagem Brasil [serial on the internet]. 2018 [cited 2020 Mar 22];17(3):190-8. Available from: https:// portalatlanticaeditora.com.br/index.php/ enfermagembrasil/article/view/1092

4. Silva MC, Ogata MN, Oliveira DC. 0 estado de arte das produções científicas nacionais das representações sociais do envelhecimento na perspectiva da saúde. Rev Kairós [serial on the internet]. 2015 [cited 2017 Aug 21];18(19):49-63. Available from: https://revistas.pucsp.br/index. $\mathrm{php/kairos/article/view/27243/19290}$

5. Guedes MBOG, Lima KC, Caldas CP, Veras RP. Apoio social e o cuidado integral à saúde do idoso. Rev Saúde Colet UEFS [serial on the internet]. 2017 [cited 2020 Mar 22];27(4):1185-204. Available from: https://www.scielosp.org/article/physis/2017. v27n4/1185-1204/pt/

6. Cabral R, Dellaroza MSG, Carvalho BG, Zani AV. 0 cuidado da pessoa idosa na atenção primária a saúde sob a ótica dos profissionais de saúde. Ciênc Cuid Saúde [serial on the internet]. 2019 [cited 2020 May 25];18(2):e45026. Available from: http:// periodicos.uem.br/ojs/index.php/CiencCuidSaude/ article/view/45026/pdf

7. Jodelet D. Representações sociais: um domínio em expansão. As representações sociais no campo da educação. In: Jodelet $D$, organizer. As representações sociais. Rio de Janeiro: EdUERJ; 2001. p. 17-44.

8. Camargo BV. ALCESTE: um programa informático de análise quantitativa de dados contextuais. Perspectivas teórico-metodológicas em representações sociais. João Pessoa: Ed. UFPB; 2005.

9. Brasil. Resolução n. 466, de 12 de dezembro de 2012 [document on the internet]. Brasília (DF): Ministério da Saúde; 2012 [cited 2017 Apr 16]. Available from: http://conselho.saude.gov.br/ resolucoes/2012/Res0466.pdf

10. Giaretta VMA, Silva AM, Renó ACM, Aguiar DAF, Arantes CMS, Posso MBS. Proposta de uma escala para avaliar o turgor da pele de idosos. Revista Ciência e 
Saúde 0n-line [serial on the internet]. 2016 [cited 2017 Aug 23];1(1):1-7. Available from: https:// revistaeletronicafunvic.org/index.php/c14ffd10/ article/view/7/12

11. Petz FFC, Crozeta K, Meier MJ, Lenhani BE, Kalinke LP, Pott FS. Pressure ulcer in intensive therapy unit: epidemiological study. Rev Enferm UFPE On Line [serial on the internet]. 2017 [cited 2018 Jan 8];11(1):287-95. Available from: https:// periodicos.ufpe.br/revistas/revistaenfermagem/ article/view/11907/14389

12. Pereira M0, Ludvich SC, Omizzolo JAE. Segurança do paciente: prevenção de úlcera por pressão em unidade de terapia intensiva. Revista Inova Saúde [serial on the internet]. 2016 [cited 2018 Jan 8];5(2):29-44. Available from: http://periodicos. unesc.net/Inovasaude/article/view/3009/2780

13. Alves CR, Costa LM, Boução DMN. Escala de Braden: a importância de avaliação do risco de úlcera de pressão em pacientes em uma unidade de terapia intensiva. Revista Recien: Revista Científica de Enfermagem [serial on the internet]. 2016 [cited 2017 Sep 15];6(17):36-44. Available from: https:// www.recien.com.br/index.php/Recien/article/ view $/ 147 / 223$

14. Silva JLG, Faustino AM. Cuidados relacionados à dermatite na área de fraldas em idosos hospitalizados. Revista de Enfermagem do Centro-0este Mineiro [serial on the internet]. 2019 [cited 2020 Mar 22];9(1):e2721. Available from: http://seer.ufsj. edu.br/index.php/recom/article/view/2721

15. Duim E, Sá FHC, Duarte YA0, Oliveira RCB, Labrão ML. Prevalência e características das feridas em pessoas idosas residentes na comunidade. Rev Esc Enferm USP [serial on the internet]. 2015 [cited 2017 Aug 21];49(1):51-7. Available from: http:// www.scielo.br/pdf/reeusp/v49nspe/1980-220Xreeusp-49-spe-0051.pdf

16. Rocha FCV, Lima M0, Gomes AV, Santos FKN, Rocha LPV. Análise da produção científica internacional sobre lesão por pressão e uso de colchões: estudo bibliométrico. Enferm Atual [serial on the internet]. 2019 [cited 2020 Mar 22];89(27):1-8. Available from: https://pt.slideshare.net/Andreadcss/revistainderme-enfermagem-atual

17. Santos JMS, Vieira BAG, Canuto PCOV, Araújo RJS, Lopes RF. Ações educativas em uma instituição de longa permanência para pessoa idosa: relato de experiência [document on the internet]. 2020 [cited 2020 May 25];3(2):1893-900. Available from: http://www.editorarealize.com.br/revistas/ cieh/trabalhos/TRABALH0 EV125 MD4 SA3 ID134 12052019185758.pdf

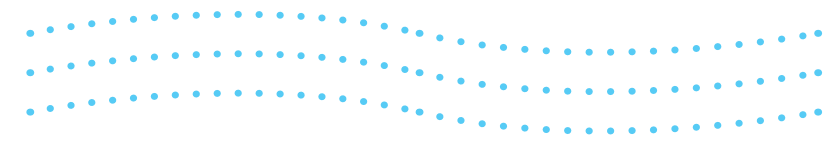

$\ldots \ldots \ldots \ldots \ldots$ .

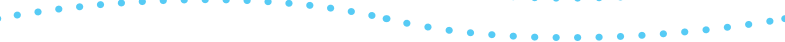

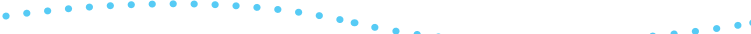
$\ldots \ldots \ldots \ldots \ldots \ldots \ldots \ldots$ $\ldots \ldots \ldots \ldots \ldots$

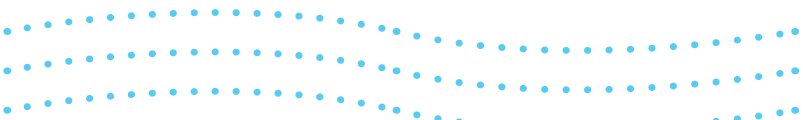

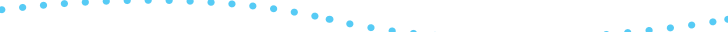
$\ldots \ldots \ldots$

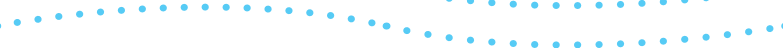

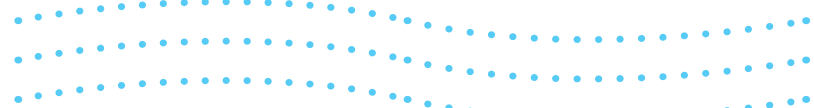

